\title{
Quantitative Microstructure Characterization of Ag Nanoparticle Sin- tered joints for Power Die Attachment
}

\author{
Yun Wang ${ }^{1,2,3}$, Jianfeng $\mathrm{Li}^{1}$, Pearl Agyakwa ${ }^{1}$, Christopher Mark Johnson ${ }^{1}$, Shuguang $\mathrm{Li}^{2}$ \\ ${ }^{1}$ Power Electronics, Machines and Control Group, The University of Nottingham, NG7 2RD, UK \\ ${ }^{2}$ Polymer Composites Research Group, The University of Nottingham, NG7 2RD, UK \\ ${ }^{3}$ China Aero-Polytechnology Establishment, Beijing 100028, China \\ E-mail: emxyw@nottingham.ac.uk
}

\begin{abstract}
The samples of sintered Ag joints for power die attachments were prepared using paste of Ag nanoparticles at 240 ${ }^{\circ} \mathrm{C}$ and $5 \mathrm{MPa}$ for 3 to 17 minutes. Their microstructural features were quantitatively characterized with scanning electronic microscopy, transmission electron microscopy, X-ray diffraction and image analysis method. The resulting normalized thickness, pore size and porosity decreased, and grain size increased with increasing the sintering time. A time dependence of the form $t^{1 / n}$ with $n$ close to 2 or 3 can be further derived for the kinetics of the thinning, densification and grain growth within the sintered Ag joints.
\end{abstract}

Keywords: Sintering of Ag nanoparticles; Power die attachment; Image analysis method; X-ray Scherrer method; Kinetics of sintering

\section{Introduction}

There is an increasing challenge in sufficient reliability for the conventional solder alloys and conductive adhesives to be used in power die attachments and interconnects for developing high power density and high performance power electronic systems. This is because the upper limit of operating temperature for Si power devices has been increased from $125^{\circ} \mathrm{C}$ towards $200^{\circ} \mathrm{C}$, and those for wide-band gap power devices (i.e. $\mathrm{SiC}$ and GaN power devices) are even higher [1]-[5]. Due to the epoxy matrix, conductive adhesives degrade at temperatures above $177^{\circ} \mathrm{C}$, and are mainly used for die attachments where conditions are below $70{ }^{\circ} \mathrm{C}$. Eutectic or near eutectic $\mathrm{Sn}-\mathrm{Ag}$ and $\mathrm{Sn}-\mathrm{Ag}-\mathrm{Cu}$ solders are more commonly used for power die attachments, but their operating temperatures are still below $125^{\circ} \mathrm{C}$ because the solders are prone to creep at elevated temperatures and the accumulation of plastic work leads to crack initiation and propagation [6]. The standard approach for high-temperature power die attachment is the use of high lead solder materials or Au-based off-eutectic solder alloys [7]. However, the Pb-containing solders will be decommissioned from high temperature electronics because of the high toxicity of lead, and the Au-based off-eutectic solders are extremely expensive and also have poor solderability [8]-[9].

To address the challenge, sintering of $\mathrm{Ag}$ particles and/or nanoparticles has been widely investigated and is promising as alternative die attachment and interconnect material and technology. In comparison with the high lead and Aubased solder alloys, Ag has a much higher melting temperature of $961.8^{\circ} \mathrm{C}$, and thus the sintered Ag joints are expected to have improved mechanical properties; in particular, improved creep resistance at elevated temperatures. The pioneering work in the sintering of Ag nanoparticles was carried out by Lu et al and Ide et al [10]-[15]. Through formulating the paste consisting of $\mathrm{Ag}$ nanoparticles and appropriate organic dispersant, binder and thinner, high quality of sintered $\mathrm{Ag}$ joints can be achieved at temperatures below $275^{\circ} \mathrm{C}$ and under pressures of a few MPa, and even without pressure. Since then, the sintering of $\mathrm{Ag}$ nanoparticles for applications in power die attachment has received more and more attention, and there is a wealthy of studies on the formulation of the Ag nanoparticle pastes, manner and pattern of printing paste, sintering process, comparison of metallization and surface topology of substrate, mechanical and thermomechanical properties, as well as thermal cycling and power cycling reliability, etc [16]-[19].

Unlike solder reflowing where solder joints are formed through liquid wetting, solid-liquid interfacial reaction and solidification, sintering is an atomic diffusion processing where the bonding is accomplished through atomic diffusion and particle consolidation. Therefore, the microstructural features of the sintered Ag joints are different from those of the reflowed solder joints, and there are re-arrangement of Ag nanoparticles, densification and reduction in porosity and grain growth occurring during the sintering process. These microstructural features in the sintered Ag joints depend on both the formulation of the paste and the sintering parameters such as heating rate, sintering temperature, pressure and time, and have effects on the thermal and electrical performance and thermo-mechanical reliability of the sintered Ag joints in service. In the existing literature [20]-[21], the values of thicknesses and porosity of the sintered Ag joints were reported. However, there was no work which has been carried out to explore the variation in those reported values and their quantitative dependence on the sintering parameters.

This present work makes an effort to quantitatively characterize the microstructural features of the sintered $\mathrm{Ag}$ joints $(13 \mathrm{~mm} \times 13 \mathrm{~mm})$ using paste of $\mathrm{Ag}$ nanoparticles for power die attachment. The microstructural features charac- 
terized include the thickness, pore size, porosity and grain size. Whenever applicable, both the average values and variations of these microstructural features are presented. Such a quantitative characterization would provide a better description to further optimize the process and predict the performance and reliability of the sintered joints used in power electronic systems. All the results obtained in the present work will not only provide new insight into understanding of the sintering process, but also be useful to guide the practical large size of sintered joint application in power die attachments.

\section{Experiments}

\subsection{Materials}

The paste of Ag nanoparticles used was obtained from Cookson Electronics (South Plainfield, NJ 07080, USA), and has been formulated with appropriate organic dispersant, binder and thinner for pressure-assisted sintering on Ag or $\mathrm{Au}$ finish. The average size of the $\mathrm{Ag}$ nanoparticles in the paste was $\sim 20 \mathrm{~nm}$.

The substrates used to attach Si devices for measuring the thickness, pore size and porosity of the sintered Ag joints were $\mathrm{Si}_{3} \mathrm{~N}_{4}$-based substrates. They were cut into $22 \mathrm{~mm} \times 17 \mathrm{~mm}$ coupons from-received $44 \mathrm{~mm} \times 34 \mathrm{~mm}$ substrates which consist of $0.32 \mathrm{~mm}$ thick $\mathrm{Si}_{3} \mathrm{~N}_{4}$ ceramic tiles sandwiched by $0.3 \mathrm{~mm}$ thick $\mathrm{Cu}$ actively brazed on both sides. The surface finish on the as-received substrates was $5 \mu \mathrm{m}$ thick NiP. An additional Ag layer of $\sim 2 \mu \mathrm{m}$ in thickness was further deposited with brush plating on the NiP finish. The Si devices attached on the $\mathrm{Si}_{3} \mathrm{~N}_{4}$-based substrates were $13 \mathrm{~mm} \times 13 \mathrm{~mm} \times 0.35 \mathrm{~mm} \mathrm{Si}$ insulated gate bipolar transistors (IGBTs) with $\sim 1 / 1 \mu \mathrm{m}$ thick Ni/Ag metallization on their back sides.

The substrates used to prepare the samples for measuring the grain size of the sintered Ag layers were $25 \mathrm{~mm} \times 15$ $\mathrm{mm} \times 0.1 \mathrm{~mm} \mathrm{Ag}$ foil substrates. After printing and drying the paste of Ag nanoparticles on the Ag foil substrates, a piece of $20 \mathrm{~mm} \times 10 \mathrm{~mm} \times 0.1 \mathrm{Fe}$ foil was placed on the top of each of the samples for applying the pressure during the final sintering stage.

\subsection{Sample preparation}

The sintered Ag samples were prepared using a house-made press. The paste of Ag nanoparticles was first printed on all the substrates with $100 \mu \mathrm{m}$ thick stainless steel stencils. In the case of the $\mathrm{Si}_{3} \mathrm{~N}_{4}$-based substrates, the size of the printed paste was $16 \mathrm{~mm} \times 16 \mathrm{~mm}$ on each of the substrates. In the case of the Ag foil substrates, the size of the printed paste was $20 \mathrm{~mm} \times 10 \mathrm{~mm}$ on each of the substrates. Then all the substrates printed with the wet paste were moved into an oven to dry at $130{ }^{\circ} \mathrm{C}$ for 30 minutes.

The thicknesses of the dried paste on the $\mathrm{Si}_{3} \mathrm{~N}_{4}$-based substrates were first measured using a Zeta-20 optical profiler (Zeta Instruments, San Jose, CA 95131) [22]. During the measurement, both the horizontal and vertical resolutions were less than $1 \mu \mathrm{m}$. Then one $\mathrm{Si}$ IGBT was placed on the central area of the dried paste on each of the $\mathrm{Si}_{3} \mathrm{~N}_{4}$-based substrates, and fixed with Kapton tape. The sample was turned upside down and put on $1 \mathrm{~mm}$ thick silicone rubber situated on the cold plate of the press. The final sintering was performed at temperature of $240{ }^{\circ} \mathrm{C}$ and pressure of $5 \mathrm{MPa}$ for $3,5,9$ or 17 minutes before releasing the pressure and cooling the sample down to room temperature within 3 minutes. Fig. 1 shows the schematic sample geometry and temperature / pressure profile used to prepare these samples.

Each of four Ag foil substrates with printed and dried Ag paste was covered by one piece of Fe foil, and then moved onto the cold plate of the press directly. The final sintering was also carried out at temperature of $240{ }^{\circ} \mathrm{C}$ and pressure of $5 \mathrm{MPa}$ for 3, 5, 9 or 17 minutes before releasing the pressure and cooling the sample down to room temperature within 3 minutes.

\subsection{Microstructure characterization}

\subsubsection{Thickness and pore}

After sintering, the samples of attaching the $\mathrm{Si}$ IGBTs on the $\mathrm{Si}_{3} \mathrm{~N}_{4}$-based substrates were mounted in epoxy resin that was cured at room temperature for 24 hours. The mounted and cured samples were first cross-sectioned with a diamond saw. Then they were successively ground with SiC papers and finally polished using diamond slurries of $3 \mu \mathrm{m}$ and $1 \mu \mathrm{m}$. The polished samples were further etched with plasma cleaner to remove the polishing-induced deformation layers prior to microstructural observation.

A Hitachi TM300 desktop scanning electronic microscope (SEM) was employed to observe the microstructure features and take images from the etched samples for measuring thickness, pore size and porosity of the sintered Ag joints. For each sample, 10 SEM images of $1280 \times 960$ pixels in resolution and $170 \times 127 \mu \mathrm{m}$ in size were taken for measuring the thickness, and another 10 SEM images of $1280 \times 960$ pixels in resolution and $17 \times 12.7 \mu \mathrm{m}$ in size were taken for measuring the pore size and porosity. All the SEM images were evenly taken along the $13 \mathrm{~mm}$ long cross section under the Si IGBT on the etched sample, and the SEM images used to measure the pore size and porosity were taken in the middle areas of the sintered Ag layer at the through-thickness direction.

All the thickness, pore size and porosity values were measured using the image analysis method which was accomplished using the Image Analysis Toolbox Version 5.0.2 of MATLAB R14SP2 (Mathworks Inc.). The key steps were to enhance the as-taken grey level SEM images and then produce the corresponding black and white binary images to extract the specific Ag layers or the pore features within the Ag layers, as detailed in the previous work [23]. Fig. 2 
presents representative SEM images and binary images used to measure the thickness, pore size and porosity values.

A data series for the thickness were obtained from the intercepted lengths of the extracted Ag layer in the throughthickness direction, which were subtracted by $2 \mu \mathrm{m}$ to account for the brush-plated Ag layer on the NiP finish, on each of the black and white binary images similar to Fig. 2b. The average value of the thickness series obtained from each binary image was calculated and used to represent the thickness at the middle point of the cross section where the SEM image was taken. The 10 thickness series obtained from the 10 SEM images were further merged as a total thickness series for the corresponding sample. The results of statistical analysis in terms of mean, standard deviation, coefficient of variation, minimum and maximum values of the total thickness series were calculated to compare the thickness and its variation among the 4 samples prepared with sintering times of 3, 5, 9 and 17 minutes.

The pore size was expressed as "apparent" radius and we calculated as:

$$
r=\sqrt{S / \pi}
$$

Where $r$ is "apparent" radius, $S$ is area of a pore measured from the black and white image similar to Fig. 2b. A data series for the pore size were obtained from each of the SEM images used to measure the pore size and porosity. Similar to thickness, the average value of the pore size series was calculated and used to represent the pore size at the middle point of the cross section where the SEM image was taken. The 10 pore size series obtained from the 10 SEM images were further merged as a total pore size series. The mean, standard deviation, coefficient of variation, minimum and maximum values of the total pore size series were calculated to compare the pore size and its variation among the 4 samples prepared with sintering times of 3, 5, 9 and 17 minutes.

The measurement of porosity is relatively simple. The porosity was calculated from the total area of the black areas in the black and white binary images similar to Fig. $2 \mathrm{~d}$. The porosity values obtained from each binary image can be used to represnt the porosity at the middle point of the cross section where the SEM image was taken. The mean, standard deviation, coefficient of variation, minimum and maximum values of the 10 porosity values obtained for each sample were further calculated to compare the porosity and its variation among the 4 samples prepared with sintering times of 3, 5, 9 and 17 minutes.

In addition, the SEM images were also taken from the surface on the as-dried paste of Ag nanoparticles, and the pore size and porosity values were measured from these SEM images as the approximate values for sintering time of zero minute.

\subsubsection{Grain size}

After the sintering, the Fe foils were not bonded on the sintered Ag layers and removed from the samples. Then the sintered Ag layers on the Ag foil substrates, as well as the as-dried paste of Ag nanoparticles, were analyzed with Xray diffraction (XRD). The XRD data were obtained using a Siemens D500 X-ray diffractometer. The experimental conditions included a $\mathrm{Cu}$ target, operating at $40 \mathrm{kV}$ and $30 \mathrm{~mA}$, and a $0.15^{\circ}$ receiving slit. The collected data angles (20) ranged from $35^{\circ}$ to $120^{\circ}$ with a step size of $0.01^{\circ}$ and a counting time of $8 \mathrm{~s}$ per step.

After obtaining the XRD data, a fundamental approach [24] was used to eliminate the machine-induced widening and calculate the sample-induced line widening at half the maximum intensity for all XRD peaks of Ag. This was done using X-ray line profile fitting program, XFIT [24], and line profile Fourier analysis, FOURYA [25], in combination with the XRD spectrum of 300 -mesh $\mathrm{LaB}_{6}$ as standard sample.

The initial intention was to estimate the grain size values of the sintered Ag layers with the William-Hall equation as expressed as [26]:

$$
\Delta K=1 / d+\alpha K^{2} c+o\left(K^{2} c\right)
$$

Where $\Delta K=2 \cos \theta \Delta \theta / \lambda$ is the integral breadth of $K=2 \sin \theta / \lambda, \theta$ is the diffraction angle, $\lambda$ is the wavelength of X-ray, $d$ is an apparen grain size, $\alpha$ is a constant, $c$ is the average dislocation contrast factor, and $o$ stands for higher order terms. However, as presneted below, the resulting data of $\Delta K$ and $K c^{1 / 2}$ were not fitted to Eq. (2) reasonably well. Thus, the Scherrer equation [27] as expressed in Eq. (3) was used to estimate the grain size values of sintered Ag layers.

$$
d=\beta \lambda /(\Delta \theta \cos \theta)
$$

Where $\beta$ is a dimensionless shape factor close to 1 and $\Delta \theta$ is the line widening at half the maximum intensity. For each sample, the grain size given below is the average vaule of 8 data calculated from the 8 peaks observed in the XRD spectrum.

In order to evaluate the grain size values estimated with the above XRD Scherrer method, the sintered Ag layers produced with sintering times of 5 and 17 minutes and the as-dried paste of Ag nanoparticles were also observed with a JEOL JEM-2000FX transmission electron microscope (TEM). The $15 \mu \mathrm{m} \times 5 \mu \mathrm{m} \times 0.2 \mu \mathrm{m}$ TEM samples were lifted out of the central areas in sintered Ag layers with a FEI Quanta 3D FIB-SEM machine. During this operation, the final accelerating voltage and current were $10 \mathrm{kV}$ and $16 \mathrm{pA}$, respectively. In addition, a few particles were obtained using a knife of stainless steel to scratch the surface of the as-dried paste of Ag nanoparticles and dispersed onto extremely thin carbon film fixed on copper mesh for the TEM observation.

\section{Results and duiscussion}

\subsection{Thickness}

SEM observation revealed that the microstrucal features were not uniform along the polished and plasma-etched cross sections of all the sintered samples. Fig. 3 shows representative SEM images taken from the sample sintered at 
$240{ }^{\circ} \mathrm{C}$ and $5 \mathrm{MPa}$ for 9 minutes. It can be seen that the sintered $\mathrm{Ag}$ layer is relatively thick and porous, and the bonding between the sintered Ag layer and both the Si IGBT and the brush-plated Ag layer on the $\mathrm{Si}_{3} \mathrm{~N}_{4}$-based substrate is poor at the edge area. By contrast, the sintered $\mathrm{Ag}$ layer is relatively thin and dense, and the bonding between the sintered $\mathrm{Ag}$ layer and both the Si IGBT and the brush-plated $\mathrm{Ag}$ layer on the $\mathrm{Si}_{3} \mathrm{~N}_{4}$-based substrate is quite good in the central area.

Figure 4 quantatively shows the variations in the thicknesses of the sintered Ag joints along the cross-sectional positions in the four samples of attaching $\mathrm{Si}$ IGBTs on the $\mathrm{Si}_{3} \mathrm{~N}_{4}$-base substrates. The sintered Ag layers at one or both edge areas are significantly thicker than those in the central areas for all the four samples. In most cases, the maximum average thickness of 70 to $80 \mu \mathrm{m}$ is thicker than two times of the minimum average thickness of 30 to $40 \mu \mathrm{m}$. Such a result reveals that there must be certain amounts of bending on the IGBTs after the sintering process.

Table 1 lists the results of statistical analysis for the total thickness data series of the sintered Ag joints in the four samples. It can be seen that the average thickness of the four sintered Ag joints, 43.5, 50.6, 59.7 and 50.3 $\mu \mathrm{m}$, fluctuate with the sintering time of 3, 5, 9 and 17 minutes. Such a fluctuatioin with the sintering time and the variations along the cross-sectional positions can be in the thickness can be attributed to the non-uniform thicknesses of the paste of Ag nanoparticles printed and dried on the substrates as evident in Fig. 5. There were also 0.1 to $0.5 \mathrm{~mm}$ wide spikes and valleys at one or both edges of the dried paste.

Therefore, the evolution of the thickness during the sintering process was explored by plotting the percentage of thickness reduction versus sintering time:

$$
P_{t r}=\frac{\left(h_{0}-h\right)}{h_{0}} \times 100 \%
$$

Where $P_{t r}$ is the percentage of thickness reduction, $h_{0}$ is the mean of the total thickness series of the as-dried paste of Ag nanopaerticles, $h$ is the mean of the total thickness series of the sintered Ag joint. The result is presented in Fig. 6 where the log-log corridinates are employed. Here and in what follows, the R value stands for the coefficient of correlation for the linear data fitting. It can be seen the percentage of thickness reduction increases, i.e the normalized thickness decreases with increasing the sintering time, and has a time dependence of the form $t^{1 / n}$ with n close to 3 .

\subsection{Pore size and porosity}

Figures 7 and 8 quantatively show the variations in the pore size and porosity of the sintered Ag joints along the cross-sectional positions in the four samples of attaching Si IGBTs on the $\mathrm{Si}_{3} \mathrm{~N}_{4}$-base substrates. Despite fluctuation with the cross-sectional positions to some extent, both the average pore size and porosity at one or both edge areas are significantly higher than those in the central area for all the four samples. Similar to the variation in the average thickness, in most cases, the maximum average pore size of 0.19 to $0.24 \mu \mathrm{m}$ and maximum porosity of $9.5 \%$ to $22.5 \%$ are close to or more than two times of the minimum average pore size of 0.11 to $0.13 \mu \mathrm{m}$ and minimum porosity of $2.5 \%$ to $9.5 \%$.

Table 1 also lists the results of statistical analysis for the total pore size data series and the porosity data series of the sintered Ag joints in the four samples. The mean values of the pore size and porosity data both decrease with increasing the sintering time. The time dependence for the reductions in the pore size and porosity are plotted in Fig. 9. Here the $\bar{r}$ is the the mean of the total pore size data series, $P$ is the mean of the porosity data series, $\overline{r_{0}}=0.17 \mu \mathrm{m}$ and $P_{0}=26.6 \%$ are the values of $\bar{r}$ and $P$ obtained from the as-dried paste of Ag nanoparticles. The reductions in the pore size and porosity have time dependence of the form $t^{1 / n}$ with $n$ close to 2 and 3 , respectively.

\subsection{Grain size}

There are 8 diffraction peaks of crystalized Ag grains which can be identified from the recorded XRD spectra of the as-dried paste of $\mathrm{Ag}$ nanoparticles and the sintered Ag samples. However, the values of $\Delta K$ and $K c^{1 / 2}$ calculated from the 8 XRD peaks cannot be not fitted to the Willimson-Hall equation satisfactorily. Figs. 10 and 11 present represtative XRD spectra and Willimson-hall plot. The poor fitting of the values of $\Delta K$ and $K c^{1 / 2}$ to the Willimson-Hall equation may be associated with the preferred orientation of $\mathrm{Ag}$ grains in all the samples. In comparison with the XRD spectrum of Ag nanoparticles reported in the existing literature [28], the relative intendsity of the (111) peaks for the present samples are all much higher, revealling preferred orientation of the Ag grains along the [111] direction. Another possibility is too wide distribution of grain size in the present samples.

Figures 12 and 13 present the TEM results obtained from the as-dried paste of Ag nanoparticles and the samples sintered for 5 and 17 minutes. It was difficult to obtain the selected electron diffraction pattern of single Ag grain from these samples, and the diffraction rings shown in Fig. 12b confirm that even single particle smaller than $100 \mathrm{~nm}$ shown in Fig. 12a contain a number of Ag grains. Furthermore, coalescence of particels and formation of necks between particles can be observed in the as-dried paste of Ag nanoparticels, and they further developed during the sintering process. The coalescence of particels and formation of necks probably ocurred along specific crystalline orientations. As a result, the preferred orientation of the Ag grains along the [111] direction was obsered from the XRD spectra shown in Fig. 10.

Nevertheless, as shown in Fig. 14, the average grain size estimated using the Sherrer Equations from the 8 XRD peaks increases with increasing the sintering time. The kinetics of the grain growth can be further derived as the the following equation:

$$
\bar{d}^{3.26}-\bar{d}_{0}^{3.26}=4.75 \times 10^{4} t
$$


Where $\bar{d}$ is the average grain size, $t$ is sintering time, and $\bar{d}_{0}$ is the value of $\bar{d}$ for the as-dried paste of Ag nanoparticles. If $d_{0}$ is assumed to be zero, a time dependence of the form $t^{1 / n}$ with $n$ close to 3 for the average grain growth can also be obtained.

\subsection{Discussion}

The non-uniform thicknesses and the spikes and valleys at the edges of the dried paste pads shown in Fig. 5 had already existed in the as-printed paste pads, and were caused by the the pulling force due to the sticking of the wet paste to the side wall of the stainless steel stencil during the printing process. As reported in a previous work [22], there was no significant change in them after the drying stage. As schematically shown in Fig. 1, the Si IGBT was reversely sited on $1 \mathrm{~mm}$ thick compliant silicone gel during the sintering stage. As a result, the non-uniformity of thicknesses and the existence of spikes and valleys at the edges of the dried paste pads would probably cause the Si IGBT to bend to some extent and relatively higher pressure applied on the Ag paste in the central areas. Unlike solder reflowing where solder joints are formed through liquid wetting, solid-liquid interfacial reaction and solidification, the sintering is a process where the bonding is accomplished through atomic diffusion and particle consolidation. The paste of Ag nanoparticles has been formulated for pressure-assisted sintering, and a higher level of pressure applied on the dried paste would promote the atomic diffusion and particle consolidation during the sintering stage. Therefore, for all the four samples of attaching the IGBTs on the $\mathrm{Si}_{3} \mathrm{~N}_{4}$-based substrates, the sintered Ag layers in the middle areas were thinner, denser and bonded to the IGBTs and substrates better than the sintered Ag layers at the edge areas.

Despite the variations in the thickness, pore size and porosity along the cross-sectional positions of the sintered Ag joints, the reductions in their average or mean values with increasing the sintering appear to have similar time exponents as shown in Figs. 6 and 9. This demonstrates that the present quantative microstructure characterization is useful to investigate their kinetics, and the thinning of the sintered Ag joints was dominated by the densification of the Ag layers during the sintering stage.

In theory for the sintering of traditional powder particles, the kinetics is generally separated into three stages: the initial stage with rearrangement of particles and densification, the intermediate stage with further densification and grain growth, and the final stage with further grain growth only [29]. As aforementioned, the average size of the Ag nanoparticles in the as-received paste was $\sim 20 \mathrm{~nm}$, and that in the as-dried paste was $29.9 \mathrm{~nm}$ (Fig. 14). This reveals that during drying the printed paste at $130{ }^{\circ} \mathrm{C}$ for 30 minutes, grain growth accompanied with the coalescence of particels and formation of necks between the particles had already started. During sintering the samples at $240{ }^{\circ} \mathrm{C}$ and 5 $\mathrm{MPa}$ for 3 to 17 minutes, the time exponent 3.26 in the presently derived Eq. (5) for describing the grain growth is in a good agreement with those value close to 3 reported for the grain growth during the intermediate stage for the sintering of traditional powder particles [30]. Also, as shown in Figs. 9 and 14, reduction in porosity and hence densification had continusously and simltaneously been progressing with the grain growth. Therefore, in addition to surface diffusion and lattice diffusion from surface due to the extremely high surface area of Ag nanoparticles, lattice diffusion from grain boundary, grain boundary diffusion and plastic deformation were also important sintering mechanisms ocurred during the sintering stage. This is because the former two are non-densifying mechanisms, and the latter three are common and possible densifying mechanisms for the present sintering of $\mathrm{Ag}$ nanoparticles.

From Figs. 9 and 14, it can be further seen that the denfication had not been completed and the Ag grains were still smaller than $100 \mathrm{~nm}$ in the sample sintered for 17 minutes. Therefore, when the sintered Ag joints are used to assemble power electronic systems, further evolution of the microstructure subjected to elevated application temperatures would occur and should be taken into account for lifetime prediction and reliability analysis. Furthermore, the final densification that can be achieved for a sintered powder compact depends on the initial contact and arrangement of the powder particles. The non-uniformity of the thicknesses and the existence of spikes and valleys in the printed pads of paste of Ag nanoparticles not only led to high variation in the thickness, pore size and porosity, but also probably had detrimental effect on achieving the final densification of the sintered Ag joints. The purpose of the present quantative microstructure characterization is to reveal the above problems. They should be able to be rectified by better control of the printing process and/or use of other methods to apply the Ag nanoparticles. For example, it is thought that replacement of the Ag paste with transferable dry film, and dispensing the Ag paste with automatic system might both be able to improve the uniformity and flattness of the Ag nanoparticle applied between the Si devices and the substrates. However, detailed investigation in these approaches needs to be carried out and this is outside the scope of the present work.

\section{Conclusions}

From the above results and discussion for the quantitative microstructure characterization of the sintered Ag joints, the following conculsions can be drawn:

1) All the thickness, pore size and porosity considerably vary along the cross-sectional positions in the sintered Ag joints, which can be attributed to non-uniform paste of Ag nanoparticles printed on the substrates;

2) In all the sintered Ag joints, the central areas with typical thicknesses of 30 to $40 \mu \mathrm{m}$ and porosity values of $2.5 \%$ to $9.5 \%$ are thinner and denser than the two edge areas with typical thicknesses of 70 to $80 \mu \mathrm{m}$ and porosity values of $9.5 \%$ to $22.5 \%$;

3) There are reductions of $23.3 \%$ to $41.5 \%$ in the avergae thickness, 0.01 to $0.03 \mu \mathrm{m}$ in average pore size and $11.4 \%$ to $20.3 \%$ in average porosity in the sintered Ag joints with increasing the sintering time from 3 to 17 minutes; 
4) The average grain size in the as-dried paste of $\mathrm{Ag}$ nanoparticles is $29.9 \mathrm{~nm}$, and those in the sintered Ag layers are 38.6, 52.4, 55.9 and $65.9 \mathrm{~nm}$ for the sintering times of 3, 5, 9 and 17 minutes;

5) The reductions in average thickness, pore size and porosity and increase in grain size exhibit similar time dependence, in the form $t^{1 / n}$ with $\mathrm{n}$ close to 2 or 3 .

\section{Acknowledgements}

The authors gratefully acknowledge the support of the Innovative Electronics Manufacturing Research Centre (IeMRC) funded by the UK Engineering and Physical Sciences Research Council (EPSRC) through research grant $\mathrm{EP} / \mathrm{H} 03014 \mathrm{X} / 1$. The authors wish to thank Oscar Khaselev and Mike Marczi of Cookson Electronics for providing the paste of Ag nanoaprticles.

\section{References}

[1] Wondrak, W., Held, R., Niemann, E., \& Schmid, U. (2001). IEEE T. Ind. Electron., 48, 307

[2] Neudeck, P. G., Okojie, R. S., \&Chen, L. Y. (2002), Proceedings of the IEEE, 90, 1065.

[3] Khan, M. A., Chen, Q., Shur, M. S., Dermott, B. T., Higgins, J. A., Burm, J., Schaff, W. J., \& Eastman, L. F. (1997), Solid-State Electron., 41, 1555.

[4] Cooper, J. A. \& Agarwal, A. (2002), Proceedings of the IEEE, 90, 956.

[5] Sugawara, Y. (2004), J. Mater. Sci., 457.

[6] Kim, H. K., Liou, H. K., \& Tu, K. N. (1995), Appl. Phys. Lett., 66, 2337.

[7] Arnold, J., McElroy, J., \&Gedney, R. Presented (2002), at JISSO/PROTEC Forum 2002.

[8] Stam, F.A., \& Davitt, E. (2001), Microelectro. Reliab., 41, 1815.

[9] Bai, J. G. ; \&Lu, G. Q. (2006), IEEE T Device. Mat. Re., 6, 436.

[10] Lu, G. Q., Calata, J. N., Zhang, Z., \&Bai, J. G. (2004), Proceedings of the IEEE CPMT Conference on High Density Microsystem Design and Packaging and Component Failure Analysis (HDP'04), 42.

[11] Zhang, Z., \&Lu, G. Q. (2002), IEEE Trans. Electron. Packag. Manuf. 25, 279.

[12] Zhang, Z., Calata, J. N., Bai, J. G., \&Lu, G. Q. (2004), 5th MPMD Global Innovations Proceedings, 129.

[13] Yamaguchi, T., Ide, E., Hirose, A., \&Kobayashi, K. F. Presented(2006), at Materials Science \& Technology 2006 Conference and Exhibition, MST\&T'06, 2, 271.

[14] Morita, T., Ide, E., Yasuda, Y., Hirose, A., \&Kobayashi, K. (2008), Jpn. J. Appl. Phys., 47, 6615.

[15] Akada, Y., Tatsumi, H., Yamaguchi, T., Hirose, A., Morita, T., \&Ide, E. (2008), Mater. Trans., 49, 1537.

[16] Silke, K., Andreas, S., \&Martin, M. (2012), Proceedings of Conference on Integrated Power Electronics Systems.

[17] Heuck, N., Palm, G., Sauerberg, T.,Stranz, A., Waag, A., \&Bakin, A. (2010), Materials Science Forum, 645.

[18] Lei, T. G., Calata, J. N., Lu, G. Q., Chen, X., \&Luo, S. (2010), IEEE T. Compon. Pack. T. , 33, 98.

[19] Ogura, H., Maruyama, M., Matsubayashi, R., Ogawa, T., Nakamura, S., Komatsu, T., Nagasawa, H., Ichimura, A., \&Isoda, S. (2010), J. Electron. Mater., 39, 1233.

[20] Navarro, L.A., Perpiñà, X., Vellvehi, M., \&Jordà, X. (2012), INGENIERÍA MECÁNICA TECNOLOGÍA Y DESARROLLO, 4, 097-102

[21] Joo, S., \&Baldwin, D. F. (2007), Proceedings of Electronic Components and Technology Conference, 219

[22] Wang, Y., Li, J.F., Agyakwa, P., Johnson, C.M., \&Li, S.G. (2014), Submitted to International Conference on Integrated Power Electronics Systems

[23] Li, J.F., Johnson, C.M., Buttay, C., Wissam, S., \&Azzopardi, S., Submitted to IEEE T. Comp. Pack. Man.

[24] Cheary, R.W., Coelho, A.A., \&Cline, J. P. (2004), J. Res.Natl. Inst. Stan., 109, 1

[25] Mileva, A., Wilsonb, M., Kannangaraa, G.S. K., \&Tran, N. (2008), Mater. Chem. Phys., 111, 346

[26] Ungár, T., \&Borbély, A. (1996), Appl. Phys. Lett., 69

[27] Ahmad, M., Mohammad, R. F., \&Mohammad, R. M.(2012), World J. Nano Sci., 2, 154

[28] Mohd, A. M. K., Sushil, K., Maqusood, A., Salman, A. A., \&Mohammad, S. A. (2011), Nanoscale Res Lett., 6.

[29] Coble, R.L.(1961), J. Appl. Phys., 32, 787-799

[30] Brook, R.J. (1976), In: Treaties on Mater. Sci. \& Tech Vol. 9, Ceramic Fabrication Processes, Wang, F. F.Y., eds, Academic Press, New York, 331 


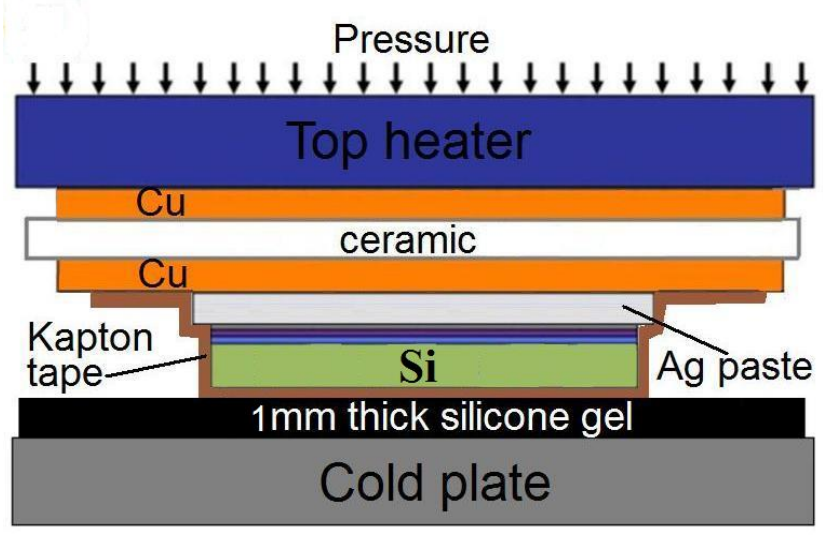

(a)

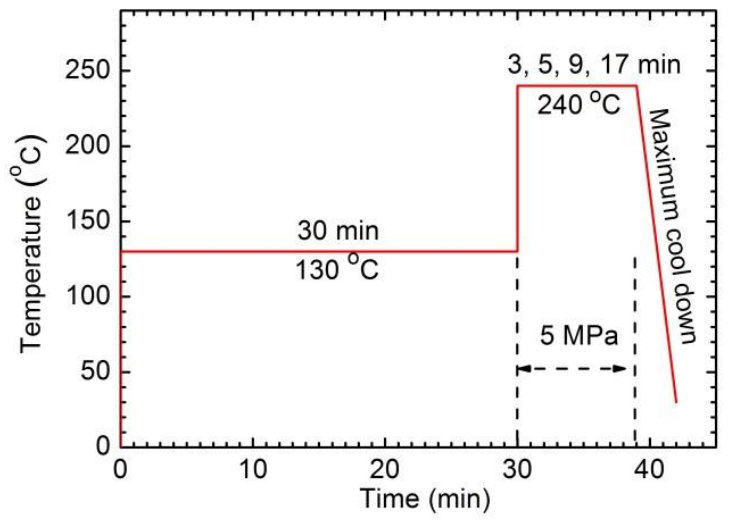

(b)

Fig. 1 Schematic diagrams of (a) sample geometry and (b) temperature and pressure profile used during the sintering process. Note that the dimensions of sample geometry are not to scale.

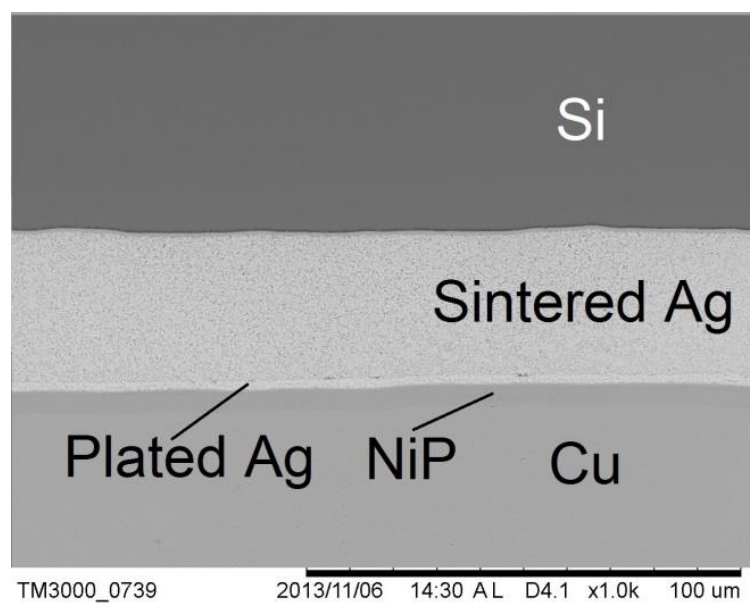

(a)

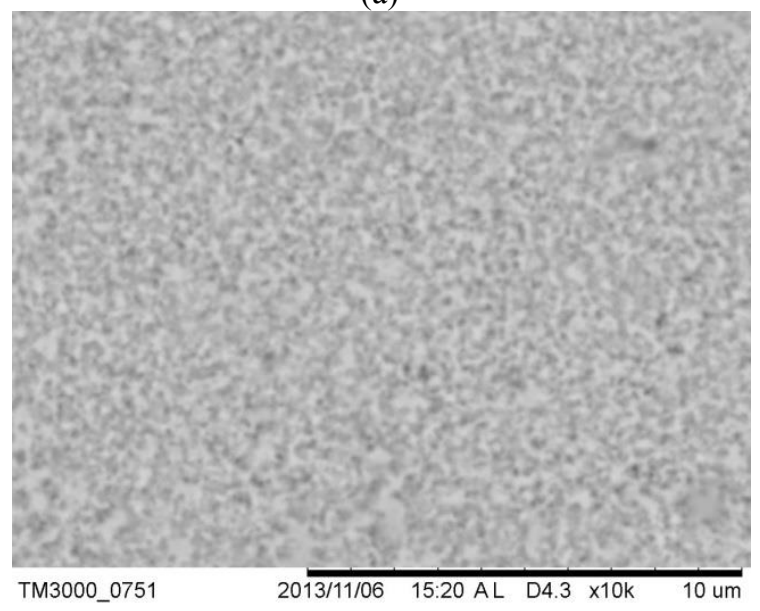

(c)
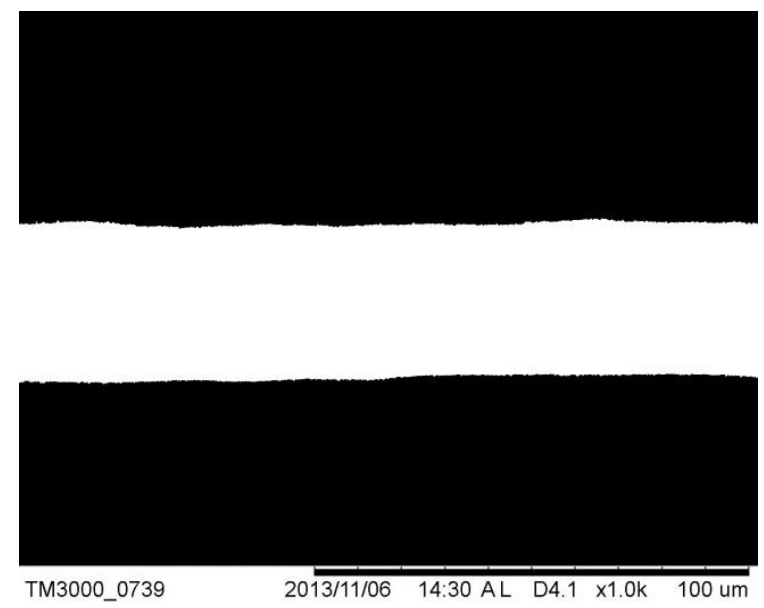

(b)

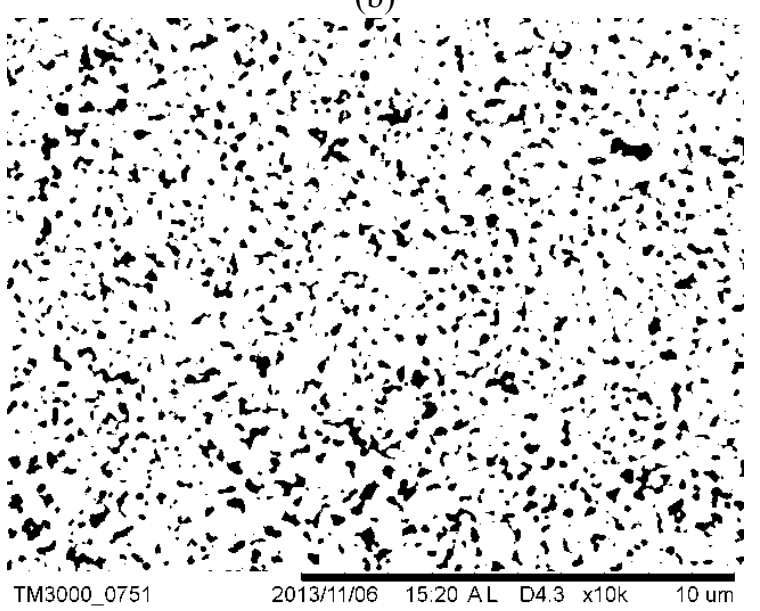

(d)

Fig. 2 (a) One representative SEM image and (b) the corresponding black and white binary used to measure the thickness values; (c) one representative SEM images and (d) the corresponding black and white binary used to measure the pore size and porosity values for the sample of attaching one Si IGBT on the $\mathrm{Si}_{3} \mathrm{~N}_{4}$-based substrate at $240^{\circ} \mathrm{C}$ and 5 MPa for 5 minutes. 


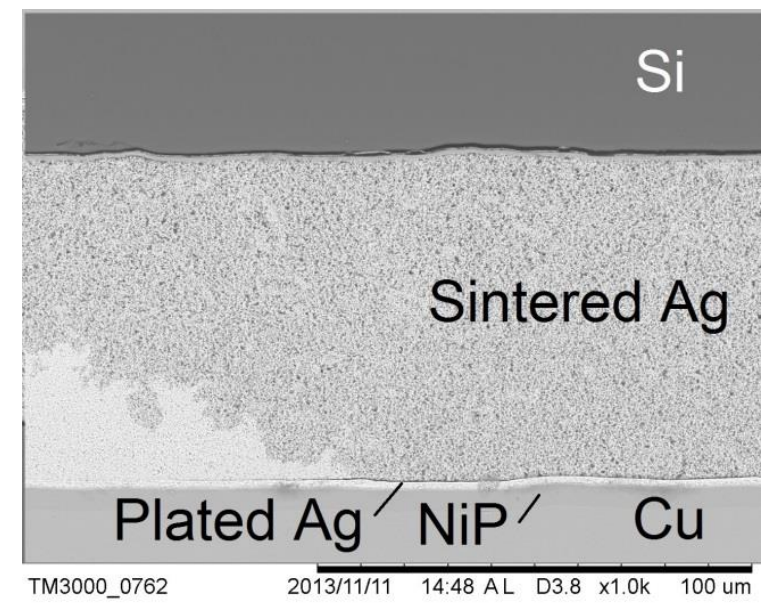

(a)

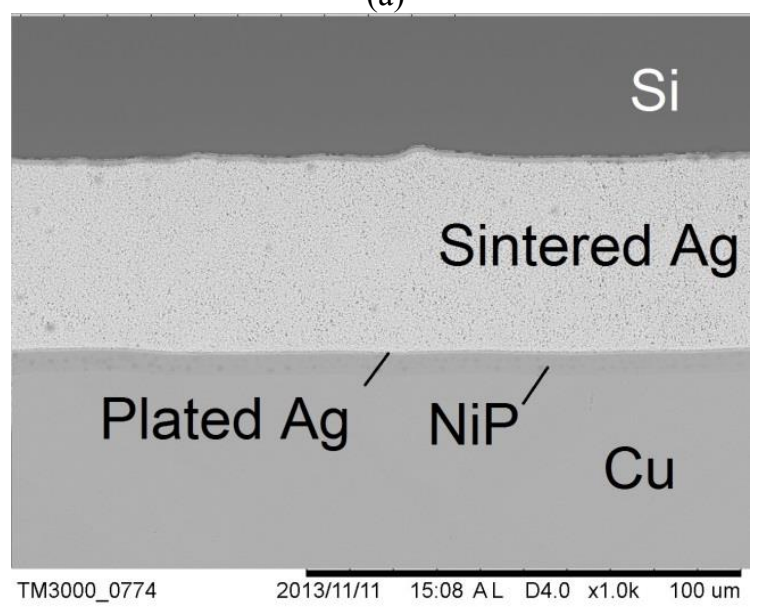

(c)

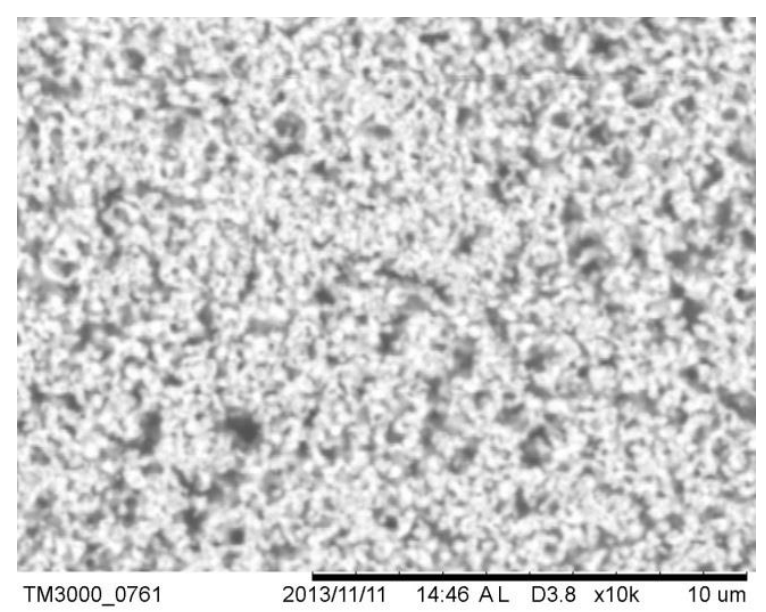

(b)

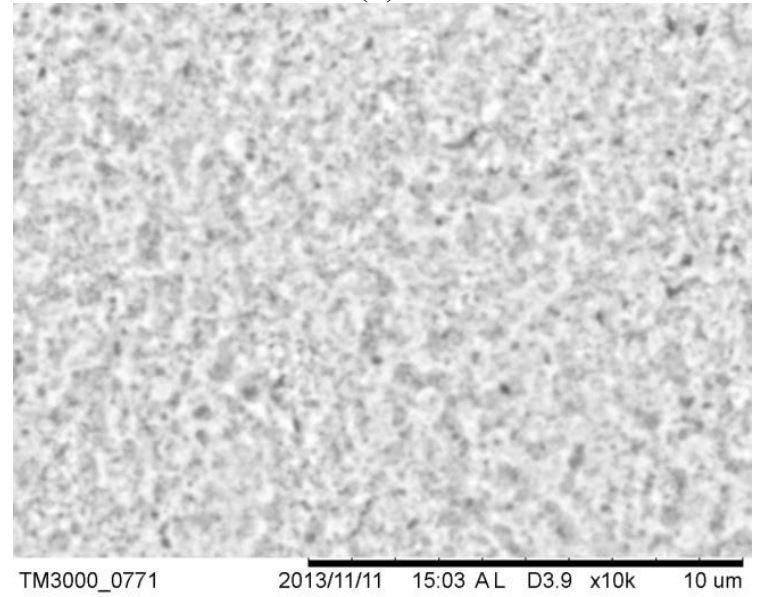

(d)

Fig. 3 SEM images taken from the polished and plasma-etched cross section of the sample of ataching one Si IGBT on the $\mathrm{Si}_{3} \mathrm{~N}_{4}$-based substrate at $240^{\circ} \mathrm{C}$ and $5 \mathrm{MPa}$ for 9 minutes: (a) overview at edge area; (b) enlarged view at edged area; (c) overview in the cenrtal area; and (d) enlarged view in the central area.

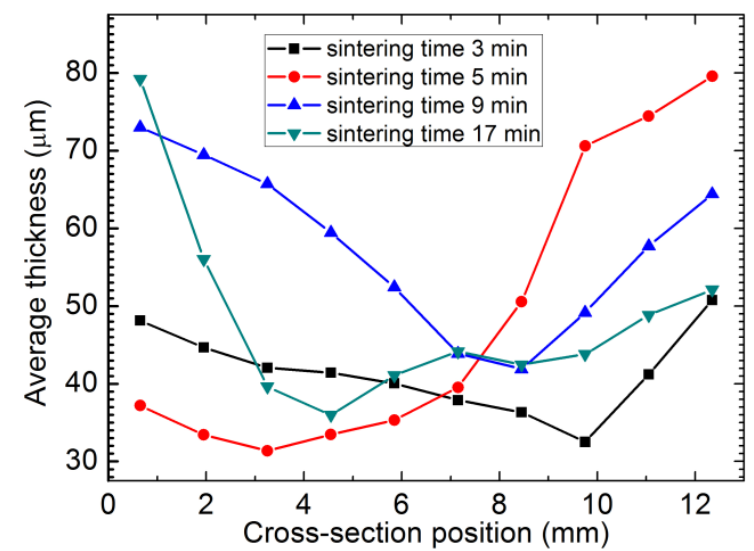

Fig. 4 Plots of thickness versus cross-sectional position for the four samples of attaching $\mathrm{Si}$ IGBTs on $\mathrm{Si}_{3} \mathrm{~N}_{4}$-based substrates. 


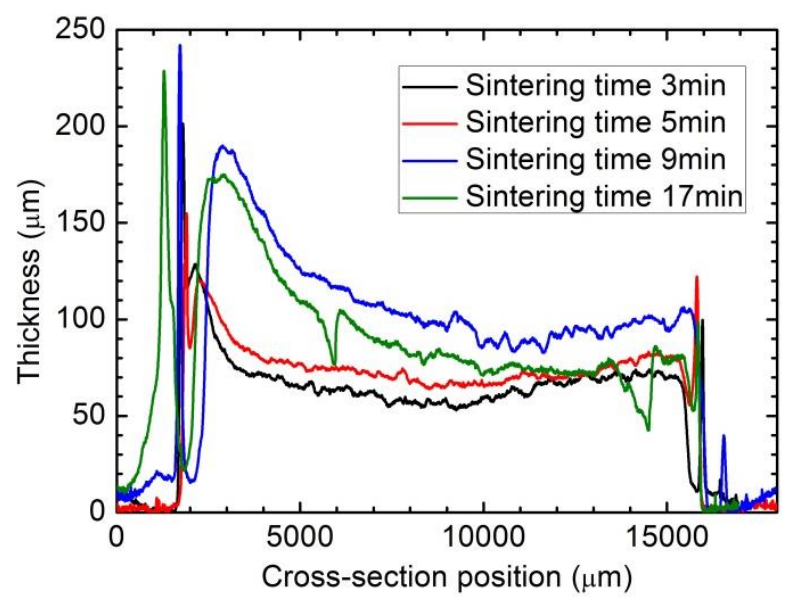

Fig. 5 Plots of thickness versus central cross-sectional position measured with a surface profiler for the four pads of paste of $\mathrm{Ag}$ nanoparticles printed on the $\mathrm{Si}_{3} \mathrm{~N}_{4}$-based substrates which were dried at $130^{\circ} \mathrm{C}$ for $30 \mathrm{~min}$ prior to attaching the Si IGBTs on them at $240{ }^{\circ} \mathrm{C}$ and $5 \mathrm{MPa}$ for 3 to 17 minutes.

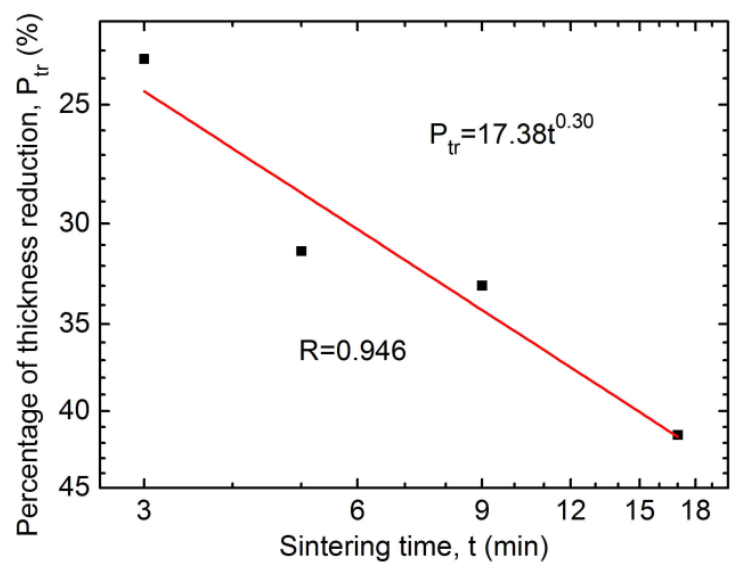

Fig. 6 Plot of percentage of thickness reduction versus sintering time on $\log -\log$ corridinates.

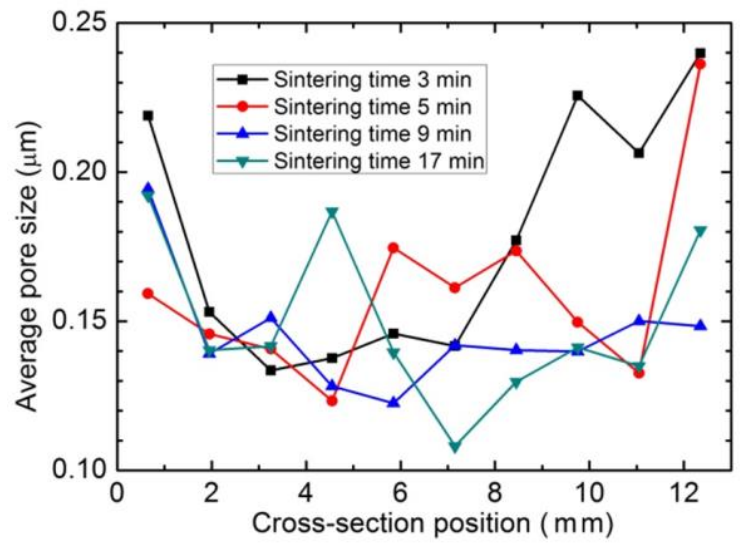

Fig. 7 Plots of average pore size versus cross-section position for the four samples of attaching Si IGBTs on $\mathrm{Si}_{3} \mathrm{~N}_{4}{ }^{-}$ based substrates. 


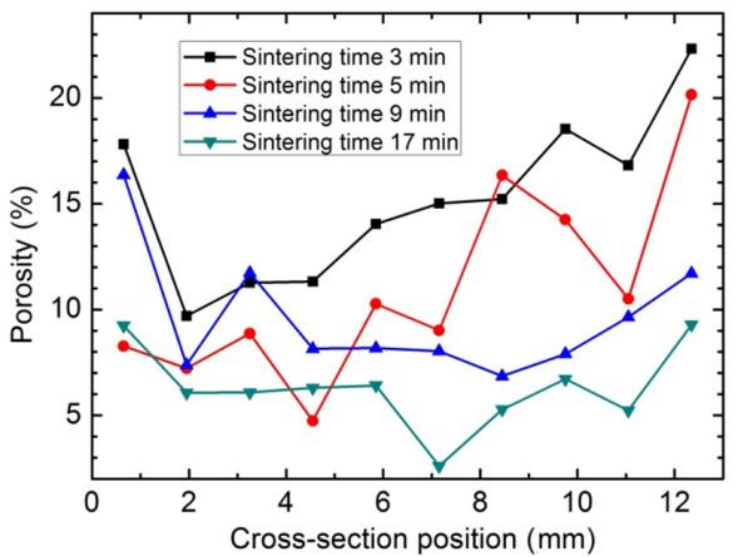

Fig. 8 Plots of porosity versus cross-section position for the four samples of attaching $\mathrm{Si}$ IGBTs on $\mathrm{Si}_{3} \mathrm{~N}_{4}$-based substrates.

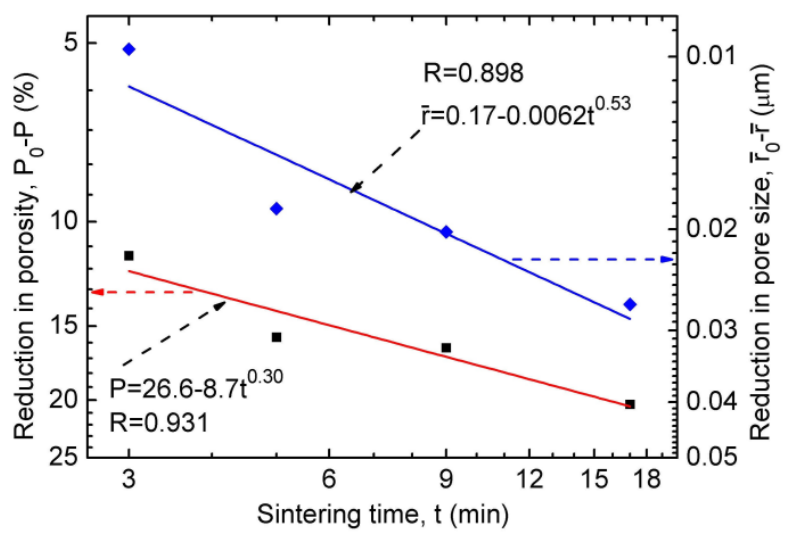

Fig. 9 Plots of reductions in pore size and porosity versus sintering time on log-log corridinates.

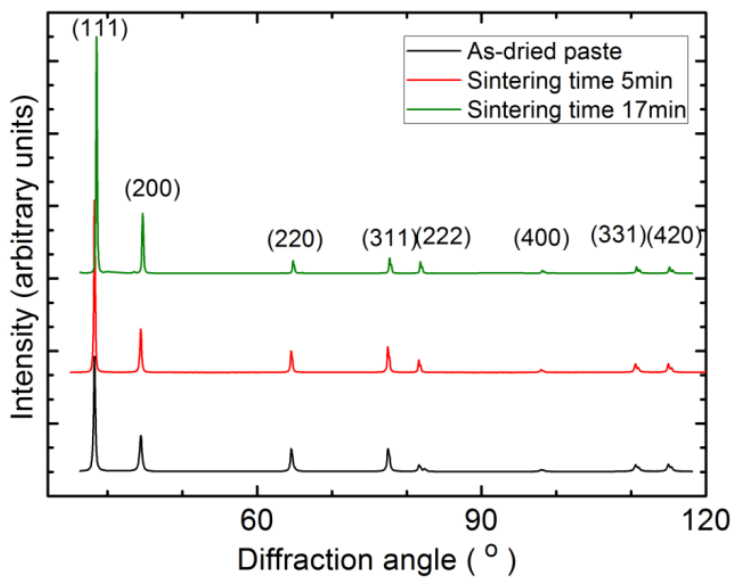

Fig. $10 \mathrm{XRD}$ spectra of the as-dried paste of Ag nanoparticles and the samples sintered for 5 and 17 minutes. 


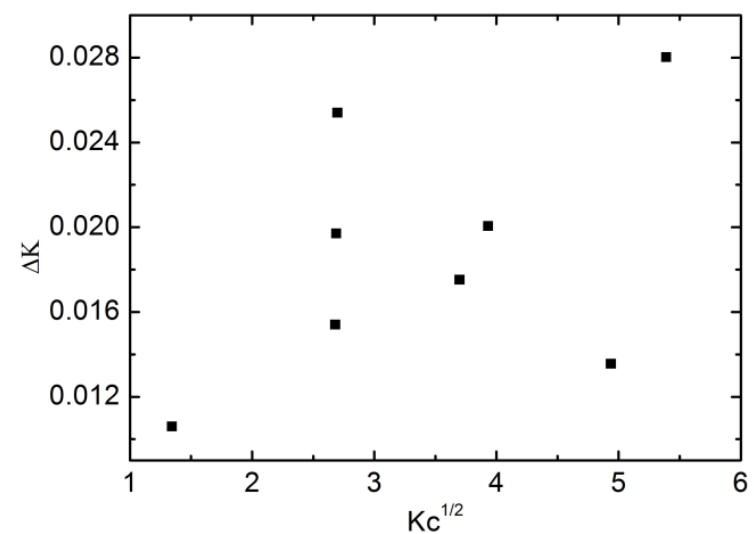

Fig. 11 Willimson-Hall plot obtained from the XRD spectrum of the sample sintered for 5 minutes.

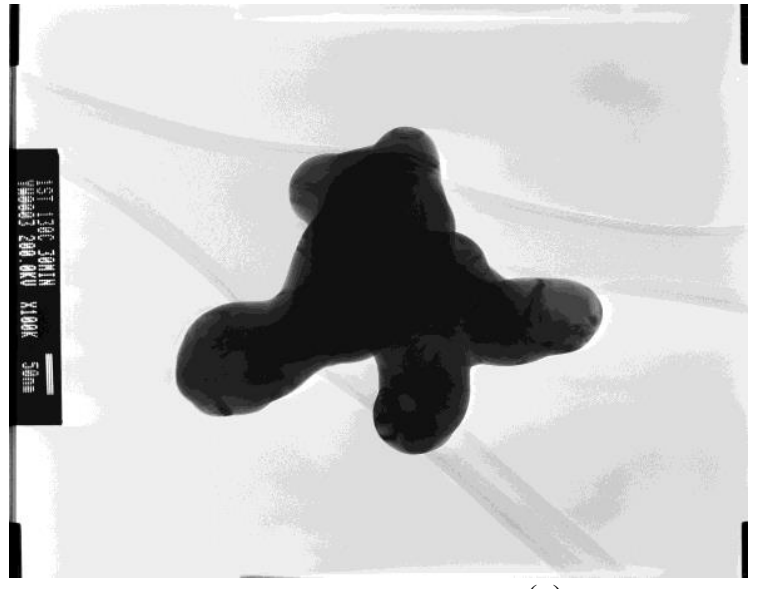

(a)

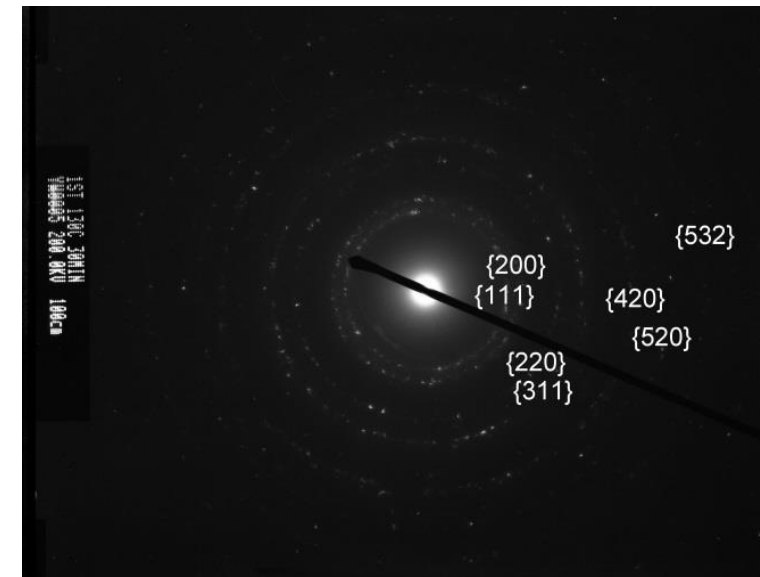

(b)

Fig. 12 TEM results of the as-dried paste of Ag nanoparticles: (a) one TEM image and (b) the corresponding electronic diffraction rings for multiple Ag grains.

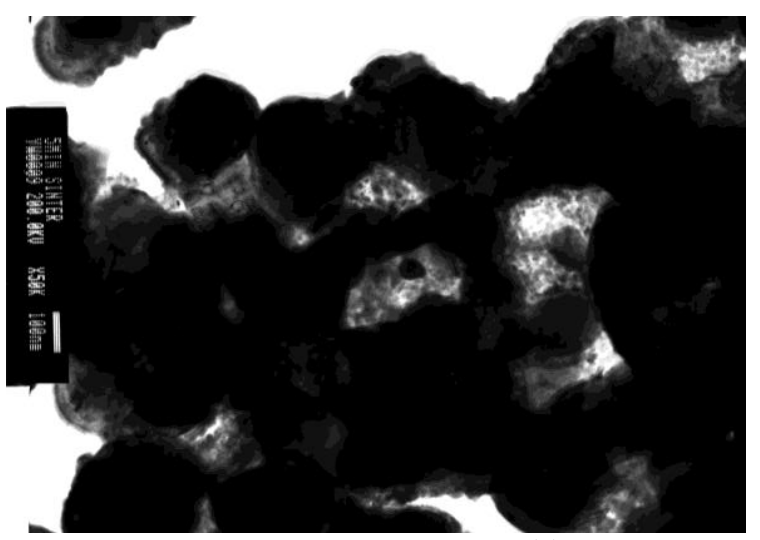

(a)

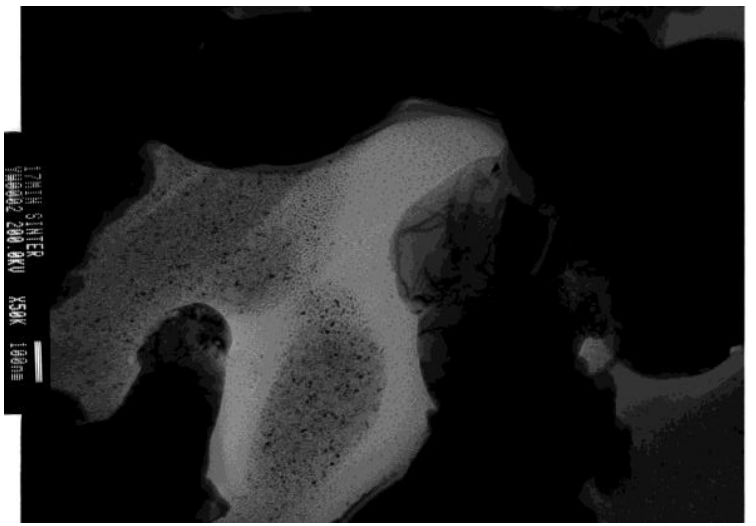

(b)

Fig. 13 TEM images taken from the samples sintered for: (a) 5 min and (b) $17 \mathrm{~min}$. 


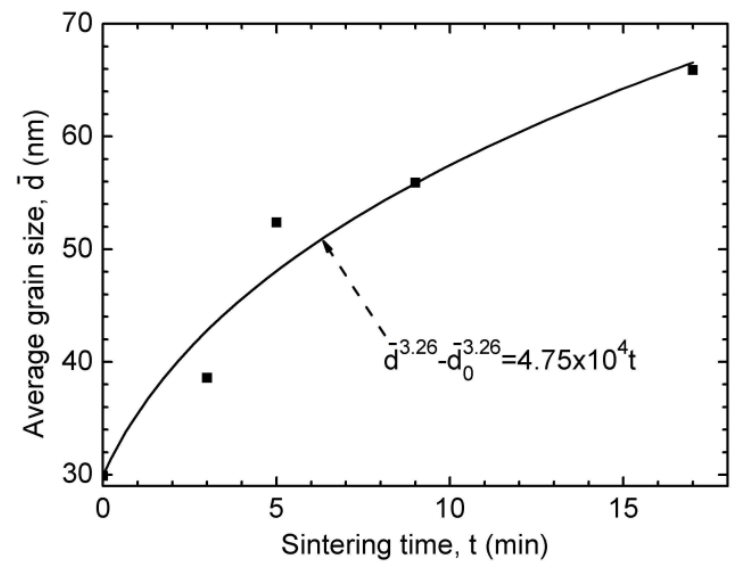

Fig. 14 Plot of average grain size versus sintering time on linear corridinates.

Table 1 The results of statistical analysis for the thickness, pore size and the porosity of the sintered Ag samples

\begin{tabular}{llllll}
\hline & & \multicolumn{4}{c}{ Sintering time (min) } \\
\cline { 3 - 6 } & & \multicolumn{1}{c}{3} & \multicolumn{1}{c}{5} & \multicolumn{1}{c}{9} & \multicolumn{1}{c}{17} \\
\hline \multirow{3}{*}{ Thickness } & Mean & 43.51 & 50.55 & 59.59 & 50.33 \\
$(\mu \mathrm{m})$ & SD & 5.19 & 18.08 & 10.50 & 11.77 \\
& CV & 0.12 & 0.36 & 0.18 & 0.23 \\
& Min & 33.33 & 32.40 & 41.96 & 36.52 \\
& Max & 55.78 & 82.87 & 77.16 & 83.53 \\
Pore size & Mean & 0.16 & 0.15 & 0.15 & 0.15 \\
$(\mu \mathrm{m})$ & SD & 0.14 & 0.11 & 0.10 & 0.09 \\
& CV & 0.87 & 0.73 & 0.67 & 0.60 \\
& Min & 0.03 & 0.03 & 0.03 & 0.03 \\
& Max & 1.51 & 1.49 & 2.22 & 0.77 \\
Porosity & & & & & \\
$(\%)$ & Mean & 15.2 & 10.97 & 10.3 & 6.3 \\
& SD & 3.85 & 4.63 & 2.92 & 1.94 \\
& CV & 0.25 & 0.42 & 0.28 & 0.31 \\
& Min & 9.69 & 4.75 & 6.85 & 2.61 \\
& Max & 22.33 & 20.15 & 16.36 & 9.28 \\
\hline SD· standard deviation; CV· coefficient & & & & &
\end{tabular}

SD: standard deviation; CV: coefficient of variation 